ARTICLE OPEN

\title{
Disintegration of simulated drinking water biofilms with arrays of microchannel plasma jets
}

Peter P. Sun $\mathbb{D}^{1,2}$ Elbashir M. Araud ${ }^{1}$, Conghui Huang ${ }^{1}$, Yun Shen ${ }^{1,4}$, Guillermo L. Monroy ${ }^{3}$, Shengyun Zhong ${ }^{2}$, Zikang Tong ${ }^{2}$, Stephen A. Boppart (iD $^{2,3}$, J. Gary Eden ${ }^{2}$ and Thanh H. Nguyen ${ }^{1}$

Biofilms exist and thrive within drinking water distribution networks, and can present human health concerns. Exposure of simulated drinking water biofilms, grown from groundwater, to a $9 \times 9$ array of microchannel plasma jets has the effect of severely eroding the biofilm and deactivating the organisms they harbor. In-situ measurements of biofilm structure and thickness with an optical coherence tomography (OCT) system show the biofilm thickness to fall from $122 \pm 17 \mu \mathrm{m}$ to $55 \pm 13 \mu \mathrm{m}$ after $15 \mathrm{~min}$. of exposure of the biofilm to the microplasma column array, when the plasmas are dissipating a power density of $58 \mathrm{~W} / \mathrm{cm}^{2}$. All biofilms investigated vanish with 20 min. of exposure. Confocal laser scanning microscopy (CLSM) demonstrates that the number of living cells in the biofilms declines by more than $93 \%$ with $15 \mathrm{~min}$. of biofilm exposure to the plasma arrays. Concentrations of several oxygen-bearing species, generated by the plasma array, were found to be $0.4-21 \mathrm{nM} / \mathrm{s}$ for the hydroxyl radical $(\mathrm{OH})$, 85-396 nM/s for the ${ }^{1} \mathrm{O}_{2}$ excited molecule, $98-280 \mu \mathrm{M}$ for $\mathrm{H}_{2} \mathrm{O}_{2}$, and 24-42 $\mu \mathrm{M}$ for $\mathrm{O}_{3}$ when the power density delivered to the array was varied between $3.6 \mathrm{~W} / \mathrm{cm}^{2}$ and $79 \mathrm{~W} / \mathrm{cm}^{2}$. The data presented here demonstrate the potential of microplasma arrays as a tool for controlling, through non-thermal disruption and removal, mixed-species biofilms prevalent in commercial and residential water systems.

npj Biofilms and Microbiomes (2018)4:24 ; doi:10.1038/s41522-018-0063-4

\section{INTRODUCTION}

Biofilms are ubiquitous in municipal and residential drinking water distribution systems, and they present significant human health concerns because of their ability to harbor pathogens ${ }^{1}$. It has long been known that biofilms are able to serve as pathogen reservoirs by supplying nutrients ${ }^{2-6}$ and shielding pathogens from disinfectants ${ }^{7,8}$. In particular, biofilms have been shown to capture and accumulate planktonic pathogens and eventually release these species into air or a water flow stream by the detachment of biofilm material ${ }^{1}$. As one example, a recent University of Virginia study ${ }^{9}$ detected, in the plumbing of several sinks, the vertical propagation of biofilm from the trap to the strainer and the subsequent dispersal of $E$. coli in the form of an aerosol. Although residual disinfectants in drinking water are mandated by the U.S. Environmental Protection Agency (EPA) for microorganism control in drinking water distribution systems, biofilms persist despite exposure to disinfectants because the extracellular polymeric substance produced by biofilms consumes the disinfectant, thereby hindering or preventing disinfectant permeation ${ }^{10,11}$.

Low temperature plasma generated in a gas flow stream is able to efficiently produce molecular radicals capable of deactivating pathogens ${ }^{12,13}$. Both the fungi and bacteria often found in tooth canals $^{14,15}$ or implanted medical devices ${ }^{12,13,16}$, for example, have been successfully treated with plasma. While the efficacy of low temperature plasma in disrupting and removing single species biofilms, and deactivating the pathogens they harbor, has been reported $^{17-20}$, little is known of the impact of plasma on the structure of the complex multi-species biofilms of municipal water systems.

We report here the disruption and erosion of biofilms, grown under simulated premise plumbing conditions, with an array of microplasma jets generated in microchannel/electrode structures fabricated by $3 D$ printing. An extensive suite of diagnostics examined the deformation and removal of the biofilms during plasma treatment, as well as the reactive oxygen species produced by the interaction of the helium (He) plasma microcolumns with room air. Specifically, optical coherence tomography (OCT) shows that the thickness of simulated water biofilms falls from a mean value of $122 \pm 17 \mu \mathrm{m}$ to $55 \pm 13 \mu \mathrm{m}$ after $15 \mathrm{~min}$. of exposure to a microplasma array operating at a modest power density $\left(58 \mathrm{~W} / \mathrm{cm}^{2}\right)$. With $20 \mathrm{~min}$. of exposure at the same power density, the biofilms vanish. Increased biofilm removal rates are readily available with larger dissipated power densities (up to $78 \mathrm{~W} / \mathrm{cm}^{2}$ in the present experiments). Furthermore, analysis of treated biofilms by confocal laser scanning microscopy (CLSM) reveals that the number of living cells remaining in the biofilm (following $15 \mathrm{~min}$. of exposure to the plasma) is reduced by $93 \%$ with respect to the control. No living cells are detected after $20 \mathrm{~min}$. of exposure of samples to the microplasma array. Concentrations of the hydroxyl radical $(\mathrm{OH})$, singlet oxygen $\left({ }^{1} \mathrm{O}_{2}\right)$, hydrogen peroxide $\left(\mathrm{H}_{2} \mathrm{O}_{2}\right)$, and ozone $\left(\mathrm{O}_{3}\right)$ produced by the microplasmas were measured by liquid chromatography or colorimetry (in the case of hydrogen peroxide), and it is these species that appear to be primarily responsible for the destruction of the biofilms and the deactivation of the pathogens they

\footnotetext{
${ }^{1}$ Department of Civil and Environmental Engineering, University of Illinois, Urbana, IL 61801, USA; ${ }^{2}$ Department of Electrical and Computer Engineering, University of Illinois, Urbana, IL 61801, USA and ${ }^{3}$ Department of Bioengineering, University of Illinois, Urbana, IL 61801, USA

Correspondence: J Gary Eden (jgeden@illinois.edu) or Thanh H. Nguyen (thn@illinois.edu)

${ }^{4}$ Present address: Department of Civil and Environmental Engineering, University of Michigan, Ann Arbor, MI 48109, USA
}

Received: 7 November 2017 Revised: 29 June 2018 Accepted: 4 July 2018

Published online: 18 October 2018 
contain. Aside from the sensitivity of the present experiments (nanomolar per second) in measuring the generation rates for hydroxyl radicals and singlet oxygen produced by the microplasmas, the primary significance of the results reported here is the demonstration of an effective tool with which the growth of biofilms in drinking water distribution networks can be mitigated. Furthermore, the insertion of microplasma arrays of cylindrical geometry into commercial or residential plumbing systems, in a fashion similar to that of conventional plumbing snakes, appears to be feasible. Such a capability will permit selective intervention into building plumbing for drinking water at locations that have resisted previous attempts at disinfection.

The approach proposed here for the control and removal of biofilms represents a significant departure from water system treatments commonly used in the U.S. which have typically involved chlorination. Through the choice of the feedstock gas and the microplasma jet characteristics (electron temperature, in particular), one is now able to specify the predominant radicals and molecular excited species generated by the plasma and its interaction with ambient air. Precisely tailoring the plasma chemistry at the point of use and matching particular plasmagenerated species with specific biofilms is now possible. The in situ application of low temperature plasma jets to biofilm control and removal has no precedent, and yet offers targeted treatment (localized and tailored to specific biofilm compositions or structures) of residential and commercial water systems.

\section{RESULTS \\ Design and performance of $9 \times 9$ microchannel plasma jet arrays}

Two diagrams of the structure adopted for the microchannel plasma jet array device are presented in Fig. 1a, b. Both are cutaway views of the monolithic design, and panel (a) of the figure illustrates the structure prior to the insertion of the electrode arrays. After entering the $4 \mathrm{~mm}$ i.d. input tube (at top, Fig. 1a), the feedstock gas (He, 99.99\%) encounters a microchannel diffuser and a shallow plenum region which serve the purpose of minimizing turbulence ${ }^{21}$ and directing gas equally to each of the square cross-section microchannels. This entire structure was fabricated in a transparent polymer by 3D printing. The electrode arrays have a multi-finger design and are machined in copper. When the electrodes are installed (Fig. 1b), this device generates a $9 \times 9$ array of microplasma columns that emerge from the lower face of the device, producing visible plumes extending approximately $1 \mathrm{~mm}$ into room air. It must be emphasized that the array design of Fig. $1 a, b$ allows for the electrode arrays to also be inserted so as to be oriented in a plane parallel to the gas flow. However, for the experiments described here, the electrodes are situated as illustrated in Fig. $1 \mathrm{~b}$.

Optical micrographs of the electrode/microchannel structure of the array are given in Fig. 1c-f. Each channel has a $400 \times 400 \mu \mathrm{m}^{2}$ square cross-section, and the images show one or more microchannels and their associated electrodes at successively greater levels of magnification. A full $9 \times 9$ array of microchannels, having an overall area of $125.4 \mathrm{~mm}^{2}\left(1.25 \mathrm{~cm}^{2}\right)$, is presented in Fig. 1c whereas only a single microchannel is shown in panel (f). The pitch (center-to-center spacing) between adjacent channels is $1.2 \mathrm{~mm}$ along both the horizontal and vertical axes of the twodimensional array which defines the areal packing density of the array as $88 \mathrm{channels} / \mathrm{cm}^{2}$. As noted earlier, all of the arrays in these experiments were constructed with a $3 \mathrm{D}$ printing tool having a spatial resolution of $50 \mu \mathrm{m}$. Panel ( $f$ ) of Fig. 1 shows that, aside from a slight "bowing" of the sidewalls of the channel, this limitation in the tool resolution has no significant adverse consequences.

Figure 2 presents time-integrated images of the visible emission produced by a $9 \times 9$ array of He plasma jets when the He mass flow rate was fixed at $0.25 \mathrm{~L} /$ minute/microchannel. Figure $2 \mathrm{a}$ is an end-on view of the fluorescence generated when the array is driven by a $20 \mathrm{kHz}$ sinusoidal voltage waveform (1.2 kV RMS), and it is clear that the jets are equally spaced along the two orthogonal coordinates of the array. The result of integrating over a $50 \mu \mathrm{s}$ window (a full cycle of the driving voltage), the intensified charge-coupled device (ICCD) camera image of Fig. 2a demonstrates that spatially uniform glow plasmas are produced within the square microchannels, but the most intense emission emanates from a cylindrical region having a diameter of 200-300 $\mu \mathrm{m}$. Images similar to that of Fig. 2a were recorded for power densities of $3.6-79 \mathrm{~W} / \mathrm{cm}^{2}$ dissipated by the array. Panel (b) is a false color image of the intensity map (derived from images such as that of Fig. 2a) acquired for a power density of $58 \mathrm{~W} / \mathrm{cm}^{2}$, and demonstrates the uniformity of the peak emission over the entire array. Two intensity lineouts of the central column of microchannel plasmas (Fig. 2c) show that the difference between the integrated intensity produced by the interior channels differs by less than $25 \%$ from that emanating from the channels at the perimeter of the array. The lineouts of panel (c) were derived from images recorded over a $500 \mathrm{~ns}$ interval centered at $t=22 \mu \mathrm{s}$ (red) or $t=48 \mu \mathrm{s}$ (blue) after the zero-crossing for the positive half-cycle of the $20 \mathrm{kHz}$ voltage waveform.

Figure $3 a$ is a side view of the optical emission recorded with a Cannon camera (Canon 5D Mark III) during the irradiation (in room air) of one of the biofilm coupons. This image illustrates vividly the interaction of the He plasma with air to produce molecular radicals, ions, and excited species. Once the microplasmas emerge into room air, the red fluorescence of the He plasma columns is transformed into blue emission as a result of the Penning ionization of the nitrogen molecule by the $\mathrm{He}\left(2^{3} S\right)$ metastable species. The B-X electronic transition of the $\mathrm{N}_{2}^{+}$ion is responsible for the violet/blue fluorescence evident above the biofilm coupon. Note that the breadth of the $N_{2}^{+}$blue emission profiles is greater than that of the He microplasmas, owing to the diffusion of $\mathrm{He}$ metastables into the cold surrounding gas.

\section{OCT and CLSM images for treated and untreated biofilms: biofilm erosion and cell deactivation}

OCT images of the biofilms were recorded in situ, both prior to exposure to the He microplasma array and at several points during the treatment process. Representative results are shown in crosssection in Fig. 3b-e where the first panel (Fig. 3b) is a profile of the film prior to plasma treatment. Figures $3 c$-e illustrate the impact on the structure and average thickness $(d)$ of the film to exposure to the plasma array for time periods $(t)$ of 5,15 , and $20 \mathrm{~min}$. Throughout these tests, the electrical power delivered to the array was fixed at 3.4 W. In each biofilm image of Fig. 3, the horizontal line indicates the boundary between the biofilm and the surface of the PVC coupon. Note that the biofilm thickness decreases from an initial value of $122 \pm 17 \mu \mathrm{m}$ (where the uncertainty represents one standard deviation) to $55 \pm 13 \mu \mathrm{m}$ after $15 \mathrm{~min}$. of exposure to the plasma array. This represents a decline in thickness of $55 \%$ and a linearized biofilm erosion rate of $4.5 \mu \mathrm{m} / \mathrm{min}$. After $20 \mathrm{~min}$. of plasma treatment, no biofilm is detectable to within experimental uncertainty. Consequently, the temporal rate of biofilm removal by the microplasma jets is decidedly nonlinear which is attributable to the time required for the radicals, and excited species, produced by the microplasma columns to diffuse through one atmosphere of air and reach the coupon.

Images similar to, and in agreement with, those of Fig. 3 but displayed in three dimensions by confocal laser microscopy (CLSM) are presented in Fig. 4. Untreated biofilms, as well as those exposed to plasma treatment for $15 \mathrm{~min}$., were stained and subsequently probed by CLSM. Panels (a) and (b) of Fig. 4 illustrate the spatial distribution of living and deactivated cells, respectively, existing in one film at $t=0$, immediately prior to the exposure of 

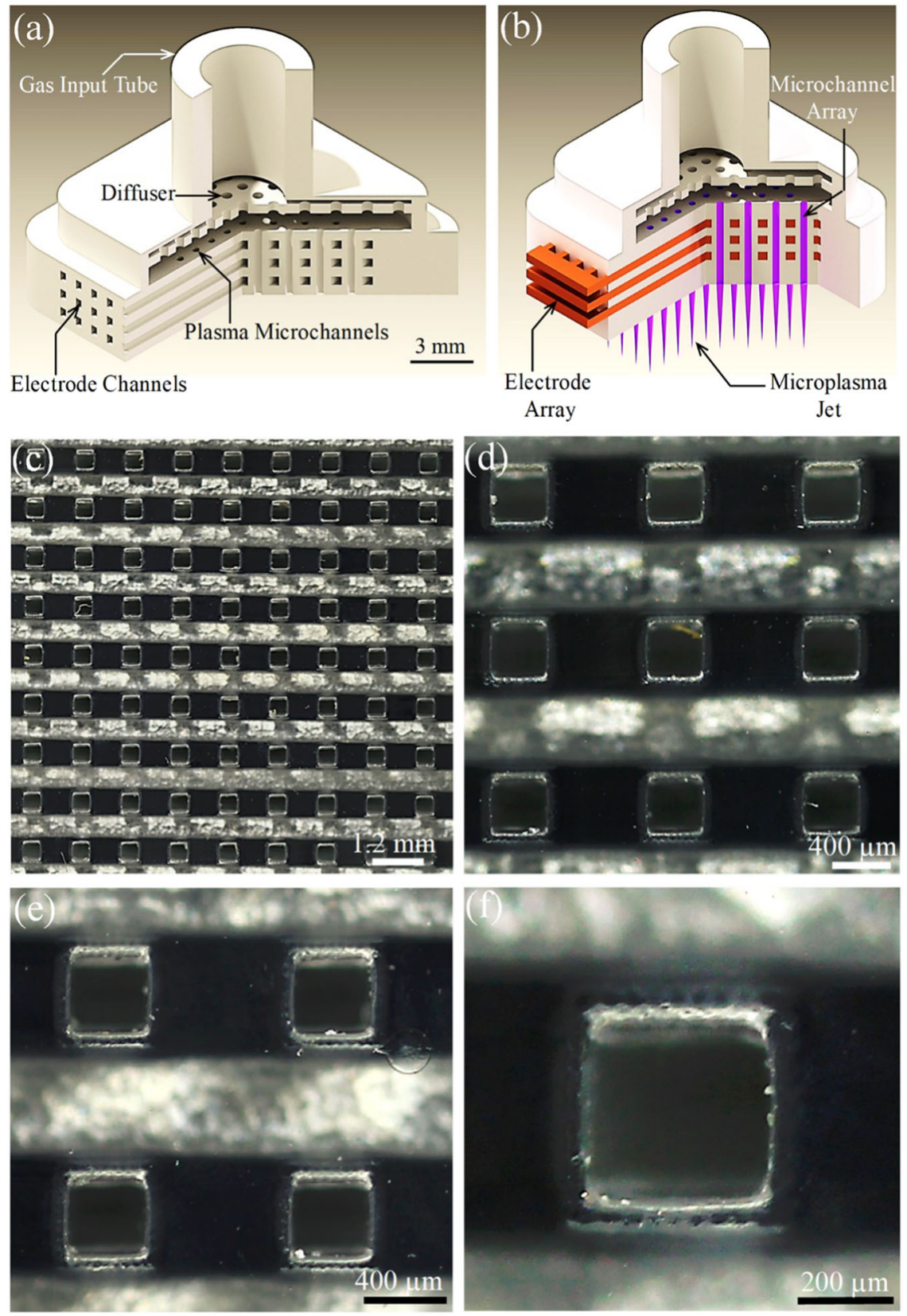

Fig. 1 Diagrams (in cutaway view) of the 3D-printed microplasma jet array devices: a monolithic structure prior to the insertion of electrodes; b illustration of the installed electrode arrays (in red) and the array in operation, producing multiple plasma jets (in blue). Feedstock gas (He in the present experiments) is injected by means of the tube at top, and the applied electric field is oriented parallel to the direction of gas flow: (see through f); Optical micrographs of a $9 \times 9$ array of square cross-section microchannels, shown at increasing levels of magnification: $\mathbf{c}$ the full $9 \times 9$ array; d a $3 \times 3$ section of the full 81 microchannel array; e a $2 \times 2$ array segment (the lower of the two horizontal lines at the bottom of all four channels is a spurious optical reflection); $\mathbf{f}$ a single microchannel, showing the spatial resolution of the 3D printing process

the biofilm to microplasma. The green fluorescence $(\lambda=500 \mathrm{~nm})$ from SYTO 9 in panel (a) denotes those cells with undamaged membranes, whereas the propidium red fluorescence $(\lambda=$ $635 \mathrm{~nm}$ ) indicates those cells that are deactivated because of ruptured or damaged membranes. After $15 \mathrm{~min}$. of exposure of this same biofilm to the microplasma array, virtually all of the living cells have been deactivated, as evidenced by the pronounced decrease (relative to that of Fig. 4a) in green fluorescence in the SYTO 9 scan of Fig. 4c. Similarly, the rapid reduction in the biomass of the film is reflected by the propidium red image of Fig. 4d which shows a dramatic decline in $635 \mathrm{~nm}$ fluorescence. Again, it must be emphasized that before $20 \mathrm{~min}$. of exposure was reached, all of the biofilms investigated vanished.

Depth-resolved studies of the green (SYTO 9) and red (propidium red) photoemission generated by CLSM were conducted for both untreated biofilms and those exposed for $15 \mathrm{~min}$. to microplasmas and the species they produce in air. Figures $4 \mathrm{e}-\mathrm{h}$ provide an overview of the images recorded in these experiments. Two-dimensional maps of the SYTO 9 (green) fluorescence are presented in Fig. $4 \mathrm{e}, \mathrm{f}$ where the upper row of images $\left(\mathrm{e}_{1}-\mathrm{e}_{4}\right)$ are those for the biofilm prior to treatment, whereas the bottom row $\left(f_{1}-f_{4}\right)$ is associated with a film subjected to $15 \mathrm{~min}$. of exposure. 

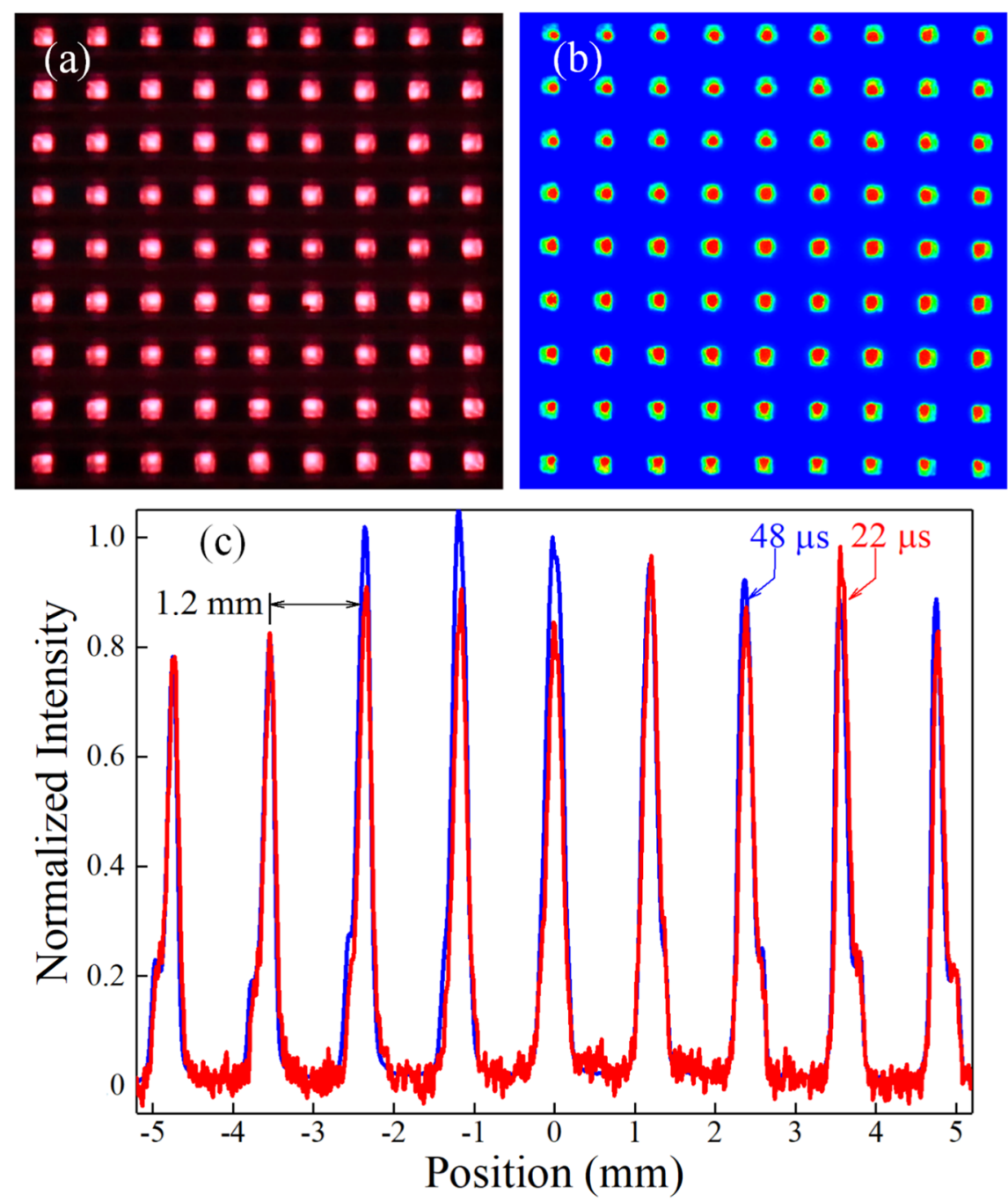

Fig. 2 End-on optical images and lineouts illustrating the uniformity of the microplasma columns emerging from the array device: a Visible emission produced from each of the 81 channels in the array when He is the feedstock gas; $\mathbf{b}$ false color image of the emission, and $\mathbf{c}$ lineouts of the array emission recorded $22 \mu \mathrm{s}$ (red) and $48 \mu \mathrm{s}$ (blue) following the zero-crossing of the positive half-cycle of the sinusoidal voltage waveform. The microchannels represented in $\mathbf{c}$ are those forming the central column in the arrays of $\mathbf{a}$ and $\mathbf{b}$. All images were recorded with a $20 \mathrm{kHz}$ sinusoidal voltage having an RMS value of $1.2 \mathrm{kV}$

For both Fig. 4e, f, images recorded at depths of $\sim 23,35,47$, and $58 \mu \mathrm{m}$ are presented. Images corresponding to those of Fig. $4 \mathrm{e}, \mathrm{f}$ but obtained by recording the spatially resolved emission from propidium red are given in Fig. $4 \mathrm{~g}$, h. Figure $4 \mathrm{i}, \mathrm{j}$ summarize the results of these experiments by illustrating the dependence of the spatially integrated SYTO 9 (or propidium red) fluorescence on depth into a biofilm. The light gray bars, derived from Figs. $4 \mathrm{e}-\mathrm{h}$ by ImageJ and COMSTAT analysis (cf. Methods), indicate the variation with depth of the integrated, normalized fluorescence intensity for the untreated biofilms, and the blue bars represent treated biofilms. On the basis of these data, one concludes that green channel (SYTO 9) fluorescence decreases by approximately $90 \%$ when the biofilms are exposed to plasma for $15 \mathrm{~min}$. Similarly, the red fluorescence (Fig. 4 j) declines by $80 \%$ during that same time period because of the rapid erosion of the biofilm by the plasma array.

After $20 \mathrm{~min}$. of biofilm exposure to the plasma array, no fluorescence-red or green-is detectable, a result consistent with Fig. 3e. Furthermore, these results have been confirmed by scanning electron microscopy (SEM) and energy dispersive spectroscopy (EDS) (Fig. 1S). The former shows no cells remaining at $t=20 \mathrm{~min}$., and EDS reveals that plasma treatment reduces carbon content in the film from 18\% (pre-treatment) to 3\% (at $\leq$ $20 \mathrm{~min}$.). The factor of six decline in carbon content and the absence of any cells underscore the conclusion that the biofilm is removed by $20 \mathrm{~min}$. of exposure. Dispersed biofilm fragments were collected and stained following the protocol of the LIVE/ DEAD BacLight ${ }^{\text {TM }}$ Bacterial Viability Kit. The images recorded by confocal laser scanning microscopy show no fluorescence (green or red), as is evident in Fig. 2S. In addition, several post-exposure samples observed by SEM showed no cells whatsoever (Fig. 3S).

\section{Production of $\mathrm{OH},{ }^{1} \mathrm{O}_{2}, \mathrm{H}_{2} \mathrm{O}_{2}$, and $\mathrm{O}_{3}$ by the microplasma jet array}

Biofilm disintegration (Fig. 3b-e) is undoubtedly the result of reactive molecular species generated by the microplasma array. Several, in particular, are capable of rupturing critical chemical bonds in the biofilm. To indirectly test this hypothesis, we quantified the concentrations of selected reactive species produced in the liquid phase by the microplasma jet array by measuring the degradation kinetics of specific probe chemicals. Experiments reported here measured the absolute concentrations of $\mathrm{OH},{ }^{1} \mathrm{O}_{2}, \mathrm{H}_{2} \mathrm{O}_{2}$, and $\mathrm{O}_{3}$, the first two of which were detected by phenol and furfuryl alcohol (FFA) solutions, respectively. Panel (a) 

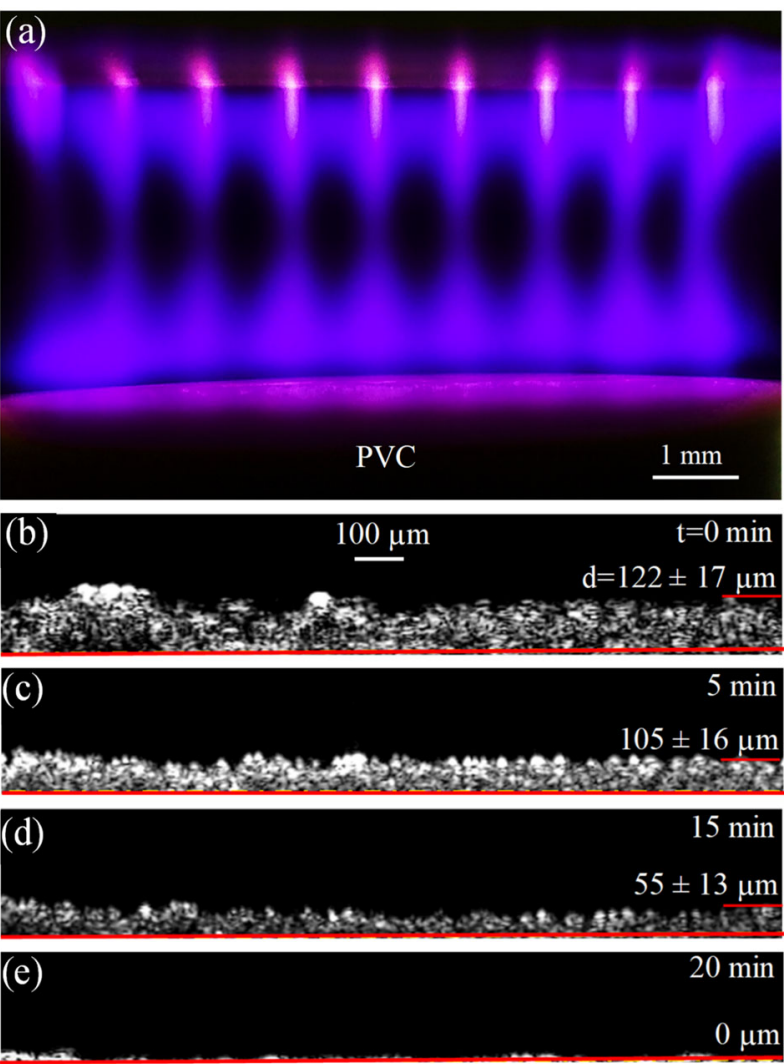

Fig. 3 a Optical image of a column of microplasma jets emerging from the array device (top) and impinging on a PVC biofilm coupon which was located $4.0 \mathrm{~mm}$ from the exit face of the array. The He gas flow was maintained at $0.25 \mathrm{~L} / \mathrm{min} /$ microchannel and the red, horizontal region indicates the surface of the coupon; b-e (in cross-section) of a biofilm, prior to and following exposure to the $\mathrm{He}$ microplasma arrays for three time periods $(t): 5,15$, and 20 min. For $t$ $=20$ min., no biofilm is detectable to within experimental uncertainty. The solid red line denotes the boundary between the biofilm and the surface of the PVC coupon

of Fig. 5 presents data showing the decay, with increasing microplasma exposure time $(t)$, of the concentration $C$ of phenol $(a$ diagnostic for $\mathrm{OH}$ ), relative to an initial value $\mathrm{C}_{0}$. Results are given for three values of power dissipated by the microplasma jet array $(P=2.4,4.9$, and $7.3 \mathrm{~W})$, and note that the ordinate is logarithmic. Measurements were recorded for array treatment times up to $t=$ $600 \mathrm{~s}$, and it is evident that the data exhibit a linear dependence on $t$ which is to be expected if the interaction of microplasma produced $\mathrm{OH}$ with phenol is a first-order process. Indeed, the solid lines in Fig. 5a represent the best fit of a pseudo-first order, rate constant kinetics model of the data and, for each value of dissipated power, the $R^{2}$ value is $\geq 0.99$. Therefore, these measurements are described well by the relation:

$\ln \left(C / C_{0}\right)=-A t+B$

where $A$ and $B$ are constants. Furthermore, the rate of decay of the phenol concentration with plasma exposure time $t$ rises with the level of power delivered to the plasma array. The rapid decline in the phenol concentration with $t$ is attributed to the formation of $\mathrm{OH}$ radicals in the PBS solution, and panel (c) of Fig. 5 shows the dependence on array power of the $\mathrm{OH}$ radical concentration, calculated from Fig. $5 \mathrm{a}$ for $t=1 \mathrm{~s}$. Note that the $\mathrm{OH}$ concentration is determined to be $0.4 \mathrm{nM}$ when $P=2.4 \mathrm{~W}$. Similarly, increasing the electrical power dissipated by the microjet array to 4.9 and $7.3 \mathrm{~W}$ raises the $\mathrm{OH}$ concentration (after only $1 \mathrm{~s}$ of plasma solution exposure) to $0.9 \mathrm{nM}$ and $2.1 \mathrm{nM}$, respectively. Further support for the premise that the microplasma jet array produces substantial concentrations of hydroxyl radicals $(\mathrm{OH})$ in the experiments of Fig. $5 \mathrm{a}$ is provided by introducing an antioxidant. Mannitol, for example, is known to be an $\mathrm{OH}$ scavenger and Fig. $4 \mathrm{~S}$ demonstrates that the addition of mannitol (0.15 M) to the PBS/ phenol solution almost completely offsets the plasma array production of $\mathrm{OH}$.

Data corresponding to those of Fig. 5a, c are presented in panels (b) and (d), respectively, for FFA/PBS solutions exposed to the microplasma jet array. In this case, FFA is a probe for the ${ }^{1} \mathrm{O}_{2}$ metastable molecule and the ${ }^{1} \mathrm{O}_{2}$ concentrations are considerably higher than those for the $\mathrm{OH}$ radical, rising from $85 \mathrm{nM}$ for $2.4 \mathrm{~W}$ of input power to the array, to $396 \mathrm{nM}$ for $7.3 \mathrm{~W}$ of power dissipated. This result is not surprising because the loss of FFA in Fig. $5 \mathrm{~b}$ is $91 \%$ after $7 \mathrm{~min}$. of exposure to the microplasmas whereas the phenol reduction in panel (a) is $84 \%$ at $t=10 \mathrm{~min}$. Recalling (cf. Methods) that the ${ }^{1} \mathrm{O}_{2}$-FFA rate constant is two orders of magnitude smaller than that for the phenol-OH interaction, it is evident that the plasma jets interacting with room air generate ${ }^{1} \mathrm{O}_{2}$ number densities considerably larger than those of the hydroxyl radical.

Figure $5 \mathrm{e}, \mathrm{f}$ summarize the results for the $\mathrm{H}_{2} \mathrm{O}_{2}$ and $\mathrm{O}_{3}$ concentration measurements, respectively, in PBS solutions when the microplasma exposure time is $t=10 \mathrm{~min}$. For $\mathrm{H}_{2} \mathrm{O}_{2}$, the measured concentration rises from $98 \mu \mathrm{M}$ at $2.4 \mathrm{~W}$ of array power $(\mathrm{P})$, to $280 \mu \mathrm{M}$ for $7.3 \mathrm{~W}$. Clearly, the hydrogen peroxide concentration rises linearly (overall) in this range of plasma array power but the preponderance of the increase occurs between $P=$ 4.9 and $7.3 \mathrm{~W}$. As shown in Fig. $5 \mathrm{f}$, the $\mathrm{O}_{3}$ concentration is sublinear in the array power despite the linear appearance of the graph (owing to the suppression of zero on the abscissa), increasing from $24 \mu \mathrm{M}$ at $P=2.4 \mathrm{~W}$ to $42 \mu \mathrm{M}$ at $7.3 \mathrm{~W}$.

For several of the molecular species of interest in Fig. 5a-f, the relative species concentration in solution is consistent with the observed dependence of the fluorescence emission intensity of a precursor excited atom, or molecule, in the gas phase. Panel $(\mathrm{g})$ of Fig. 5 is a low resolution $(\Delta \lambda \approx 0.3 \mathrm{~nm})$ spectrum of the spontaneous emission from the $9 \times 9$ microjet array, recorded over the $300-850 \mathrm{~nm}$ wavelength region. Dominated by the $\mathrm{N}_{2}$ $(\mathrm{C} \rightarrow \mathrm{B})$ electronic transition that is responsible for molecular emission at 337.1, 357.7, and $380.5 \mathrm{~nm}$, for example, this spectrum also shows contributions from the singly charged nitrogen ion ( $\mathrm{N}_{2}^{+}$), atomic oxygen, and $\mathrm{OH}$. Furthermore, the linear dependence of the $\mathrm{OH}(\mathrm{A} \rightarrow \mathrm{X})$ fluorescence intensity on the plasma array voltage, observed in Fig. $5 \mathrm{~h}$, is reflected in the variation of the $\mathrm{OH}$ and $\mathrm{H}_{2} \mathrm{O}_{2}$ concentrations (in solution) with the power dissipated by the microplasma array. Similarly, the nonlinear rise of the ${ }^{1} \mathrm{O}_{2}$ concentration with increasing power delivered to the array (Fig. $5 d$ ) is to be expected because the population of the $3 p{ }^{5} P_{2}$ state of atomic oxygen (upper level of the $777 \mathrm{~nm}$ transition of Fig. $5 \mathrm{~g}, \mathrm{~h}$ ) also increases in a nonlinear manner with rising plasma voltage (and power). Owing to its internal energy of $10.7 \mathrm{eV}$, the $\mathrm{O}\left(3 \mathrm{p}^{5} \mathrm{P}_{2}\right)$ state is a potential precursor to ${ }^{1} \mathrm{O}_{2}$ in both the gas and solution phases.

\section{DISCUSSION}

Exposure of simulated drinking water biofilms to the excited species, radicals, and ultraviolet radiation produced by a $9 \times 9$ array of microplasma jets is found to erode (and remove) the films, as well as deactivate the cells they contain. Measurements of biofilm thickness by OCT demonstrate that $122 \mu \mathrm{m}$ thick films are removed entirely by $20 \mathrm{~min}$. of exposure to the plasma array. Combined with the observation that the number of living cells in the biofilms falls by $>93 \%$ with $15 \mathrm{~min}$. of exposure (and reaches zero by $t=20 \mathrm{~min}$.), these results indicate that arrays of low temperature plasma jets offer an inexpensive and nonthermal tool 
with which the growth of biofilms in drinking water distribution systems can be mitigated or eliminated.

A few additional comments regarding the applicability of this technology to residential or commercial water networks are warranted. Although the geometry of the microplasma array of Fig. 1 was chosen for experimental convenience, it is clearly not suitable for implementation in drinking water distribution systems. However, multiple configurations of microplasma jet arrays are currently available. One of these is the cylindrical geometry, described in U.S. Patent No. 8,957,572, in which microplasma jets propagate radially outwards from a central cylinder having a diameter that can be less than $6 \mathrm{~mm}\left(1 / 4^{\prime \prime}\right)$. Furthermore, the
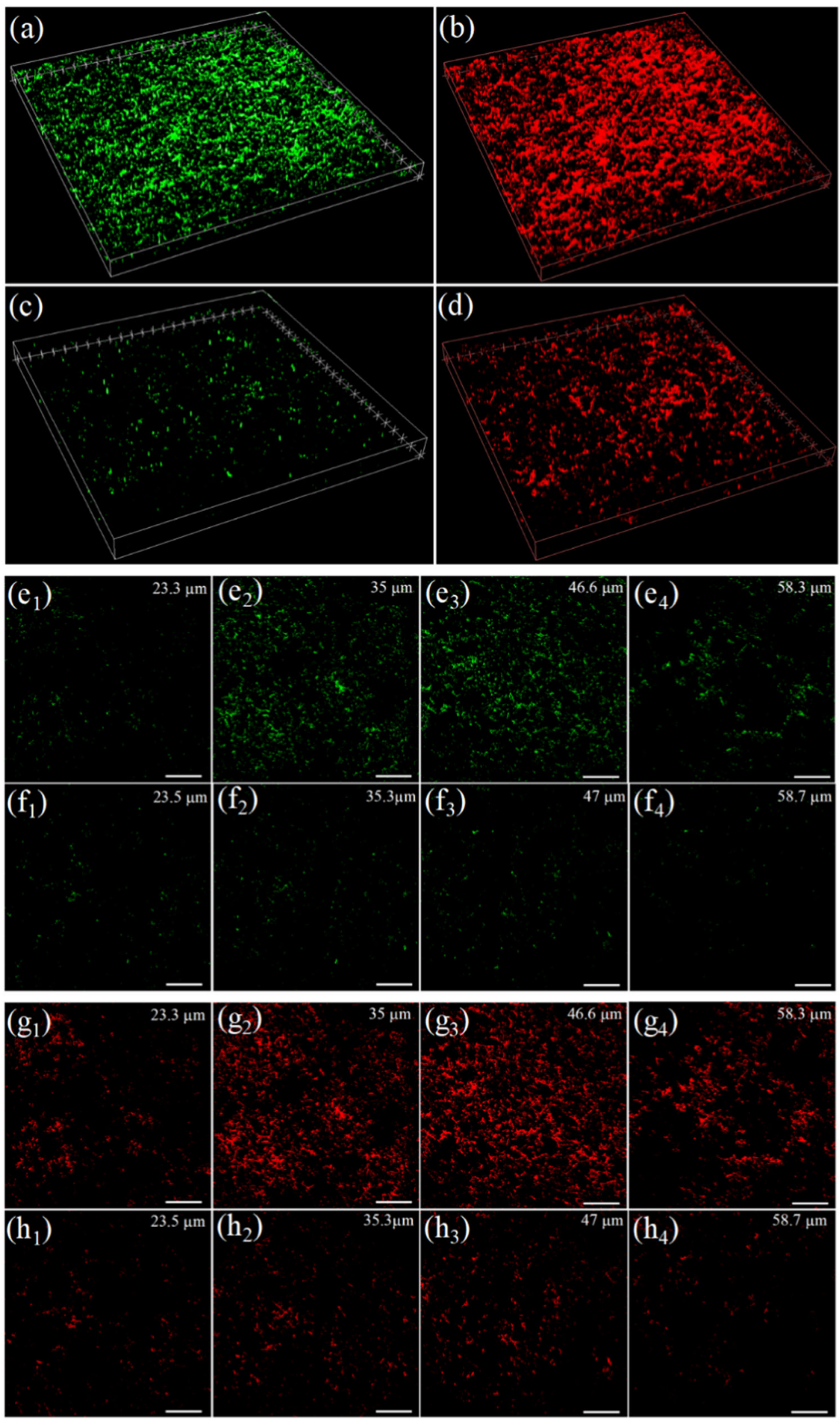


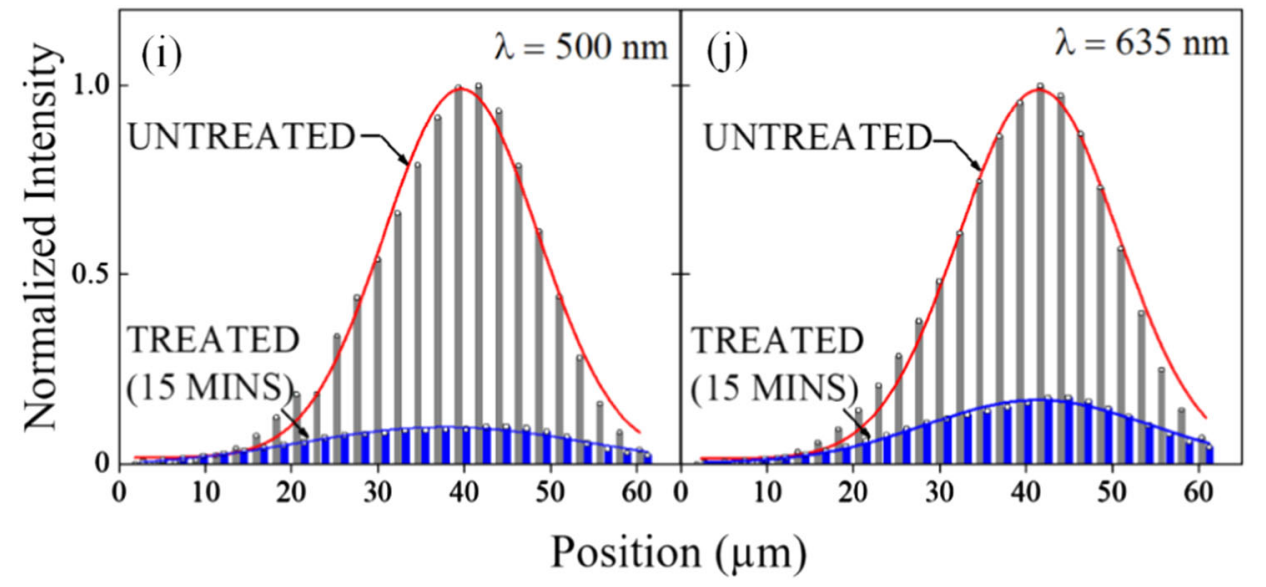

Fig. 4 CLSM images of the biofilm of Fig. 3, recorded prior to and after exposure of the film to the microplasma array: $\mathbf{a}, \mathbf{b} 3 \mathrm{D}$ images of the biofilm at $t=0$ (immediately prior to microplasma treatment), indicating the distribution of living cells (panel a, SYTO 9 fluorophore, $\lambda=$ $500 \mathrm{~nm}$ ) and deactivated cells (b, propidium red fluorophore, $\lambda=635 \mathrm{~nm}$ ); $\mathbf{c}, \mathbf{d}$ CLSM images corresponding to those of $\mathbf{a}$ and $\mathbf{b}$, respectively, but recorded after 15 min. of treatment of the biofilm with low temperature plasma; $\mathbf{e}-\mathbf{h}$ Series of CLSM images recorded for SYTO 9 (green, $\lambda$ $=500 \mathrm{~nm})$ staining, at several values of depth into a biofilm $\left(\mathbf{e}_{1}-\mathbf{e}_{4}, \mathbf{f}_{1}-\mathbf{f}_{4}\right)$. The row of images at top $\left(\mathbf{e}_{1}-\mathbf{e}_{4}\right)$ were obtained prior to treatment of the biofilm with plasma, while those in the lower row $\left(\mathbf{f}_{1}-\mathbf{f}_{4}\right)$ were acquired after $15 \mathrm{~min}$. of exposure to the microplasma array. The white horizontal bars in each panel represent a length of $200 \mu \mathrm{m} ; \mathbf{g}_{1}-\mathbf{g}_{4}, \mathbf{h}_{1}-\mathbf{h}_{4}$ : Images corresponding to those of Fig. $4 \mathbf{e}_{1}-\mathbf{e}_{4}$ and $\mathbf{f}_{1}-\mathbf{f}_{4}$ but recorded by mapping the fluorescence from propidium red $(\lambda=635 \mathrm{~nm})$. Again, the horizontal bars represent a length of $200 \mu \mathrm{m}$; $\mathbf{i}$, $\mathbf{j}$ : Summary of the green (SYTO $9, \lambda=500 \mathrm{~nm}$, panels $\mathbf{e}$ and $\mathbf{f}$ ) and red (propidium red, $\lambda=635 \mathrm{~nm}$, panels $\mathbf{g}$ and $\mathbf{h}$ ) fluorescence depth profiles, measured for biofilms that were untreated (light gray bars) and those treated by exposure to the microplasma jet array for 15 min. (dark blue bars). The red curves represent the fitting of a Gaussian to the data

plasma microcolumns are produced in an azimuthally symmetric pattern and are spaced equally along the axial coordinate of the array. The length of such devices can be extended so as to increase the surface area being treated at any given time, thereby expediting the treatment process. Such cylindrical devices may be introduced to premise plumbing systems in a manner similar to that for conventional plumber "snakes" which typically include integrated CCD cameras. The simultaneous insertion of several snakes will accommodate portions of the plumbing system having copper or PVC pipe of differing diameters, thus decreasing treatment time.

Figure 6 presents several diagrams, reproduced from Ref. ${ }^{22}$, illustrating the structure of a biofilm treatment system comprising multiple cylindrical microplasma jet arrays integrated with a modified plumbing snake. The inset of Fig. 6 is a qualitative drawing (not to scale) of a cylindrical microjet array having a diameter sufficiently small so as to allow insertion of the array into the snake. Feedstock gas (which need not be helium) flows to each cylindrical array along the axis of the snake, which also accommodates electrical connections providing power to the jet arrays and a camera.

For the current electrical efficiency of the process described here, biofilm control or removal with microplasma jet arrays is economically viable. Assuming the complete removal of biofilm in $20 \mathrm{~min}$. at an average power density of $60 \mathrm{~W} / \mathrm{cm}^{2}$, the electrical power required is $0.02 \mathrm{kWh}$ per $\mathrm{cm}^{2}$ of surface area (lower if complete biofilm removal is not necessary). Consequently, even if one assumes that engineering development of these arrays does not result in increased plasma generation efficiency and reduced treatment time, the cost of power associated with treating $(2-10) \times 10^{5} \mathrm{~cm}^{2}$ of surface area is not prohibitive. Because the plasma treatment of biofilms on the interior surface of water pipes necessarily confines the molecular radicals and excited species generated by the microplasmas, it is expected that biofilm removal rates in water distribution systems will be significantly larger than those reported here for the present (planar) geometry. As is evident in Fig. 3a, the present geometry allows radicals to escape along any plane transverse to the axes of the microplasma jets.
This technology appears to be of particular value for intervention in those circumstances in which residential or commercial plumbing is known to have been compromised by one or more pathogens, and previous and persistent efforts to purge or disinfect the system have proven unsuccessful. Arrays of microplasma jets provide a chlorine-free and in situ tool for controlling biofilms and pathogen populations with a degree of specificity (with regard to spatial localization and customized treatment of various biofilms) not available previously.

\section{METHODS}

\section{Preparation of simulated drinking water biofilms}

Biofilms were grown on polyvinyl chloride (PVC) coupons from a groundwater source of drinking water in Urbana-Champaign, Illinois (USA). Treated by a greensand filter, this water was found to contain $1.65 \pm$ $0.08 \mathrm{mM} \mathrm{Ca}^{2+}, 1.16 \pm 0.01 \mathrm{mM} \mathrm{Mg}^{2+}$, and $1.04 \mathrm{mM} \mathrm{Na}^{+}$, and its measured hardness is $281 \pm 8 \mathrm{mg} / \mathrm{L}$. The total organic content (TOC) of the groundwater is $1-1.6 \mathrm{mg} / \mathrm{L}$, and the $\mathrm{pH}$ is in the range of 7.5-7.8. Because the process for growing biofilms has been described in detail previously $^{23,24}$, it will be only briefly reviewed here. PVC coupons (RD 128-PVC, BioSurface Technologies Corporation, Bozeman, Montana) served as the substrate for the biofilms. After placing the coupons into $\mathrm{CDC}$ reactors (CBR 90-2, Biosurface Technologies Corporation), groundwater was pumped continuously into the reactors and biofilm development took place under shearing conditions because of continuous stirring within the reactors at $125 \mathrm{rpm}$, which corresponds to an Re value of 2384 . No extraneous nutrients or microorganism strains were introduced to the reactors, and the biofilms were allowed to develop undisturbed for 10 months prior to the characterization and plasma treatment experiments.

\section{Plasma treatment of biofilms}

All of the experiments reported here were conducted with the microplasma array of Fig. 1, driven by a $20 \mathrm{kHz}$ sinusoidal waveform having an RMS value of $1.2 \mathrm{kV}$. Helium gas flow was maintained at $0.25 \mathrm{~L} /$ $\mathrm{min} /$ microchannel (approximately $20 \mathrm{l} / \mathrm{min}$. total flow rate) and the exit face of the array was situated $4 \mathrm{~mm}$ from a PVC coupon onto which a biofilm had been grown. In total, seven biofilm coupons were examined in this study. 

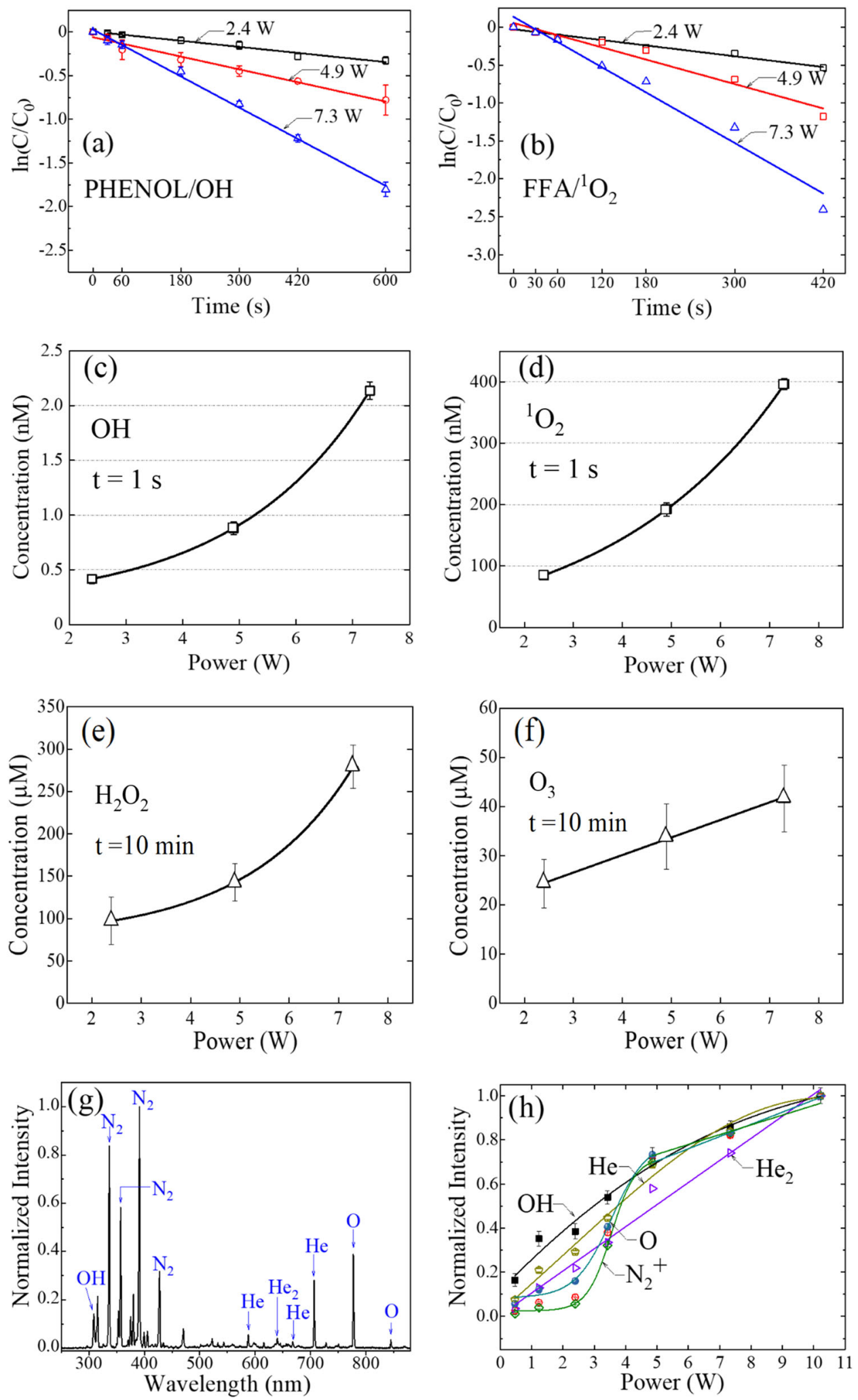

Biofilm imaging by optical coherence tomography, confocal laser scanning microscopy, scanning electron microscopy, and energy dispersive spectroscopy

All biofilms were examined in situ, both prior to and after plasma treatment, by $\mathrm{OCT}^{25,26}$. A custom built, spectral domain OCT system, emitting low-coherence light with a central wavelength of $1322 \mathrm{~nm}$ and a bandwidth of $106 \mathrm{~nm}$, offers an axial and transverse resolution of $4.2 \mu \mathrm{m}$ and $3.9 \mu \mathrm{m}$, respectively, in $\mathrm{air}^{27}$. For each biofilm sample, cross-sectional images having a volume of $3.1 \mathrm{~mm}$ (transverse dimension) $\times 2.1 \mathrm{~mm}$ (depth) $\times 4 \mathrm{~mm}$ were recorded at three locations on the film, and 600 
Fig. 5 a-d Concentrations of $\mathrm{OH}$, and ${ }^{1} \mathrm{O}_{2}$ produced by a He microplasma jet array in phenol or FFA solutions: a Decay of the phenol concentration, $C$ (relative to its initial value $C_{0}$ ), for microplasma exposure times up to $600 \mathrm{~s}$. Data are presented for several values of power delivered to the $9 \times 9$ array, and note that the ordinate is logarithmic; $\mathbf{b}$ Data similar to those of a but for FFA in a PBS solution; c Concentration of $\mathrm{OH}$, calculated from the data of $\mathbf{a}$, for three values of power dissipated by the plasma; d Dependence on microplasma array power of the ${ }^{1} \mathrm{O}_{2}$ concentration (calculated from $\mathbf{b}$ ). The results of panels $\mathbf{c}$ and $\mathbf{d}$ are those recorded after only one second of exposure of the phenol/PBS or FFA/PBS solutions to the plasma; e-f: Measurements of the concentrations of: $\mathrm{H}_{2} \mathrm{O}_{2}$, and $\mathrm{O}_{3}$ after 10 min. of plasma array exposure to PBS solutions. Data are given for three values of power $(P)$ dissipated by the microplasma jet array; $\mathbf{g}$ Emission spectrum of the $9 \times 9$ microplasma jet array, recorded end-on over the $300-850 \mathrm{~nm}$ spectral interval. The He backing pressure for the jets was 785 Torr ( $300 \mathrm{~K}$ pressure), the driving voltage was a $20 \mathrm{kHz}$ sinusoid, and the array emerged into room air; $\mathbf{h}$ Variation with peak driving voltage of the normalized fluorescence generated by several selected emitters. The oxygen atomic transition is the $777 \mathrm{~nm}$ line of panel $\mathbf{g}$

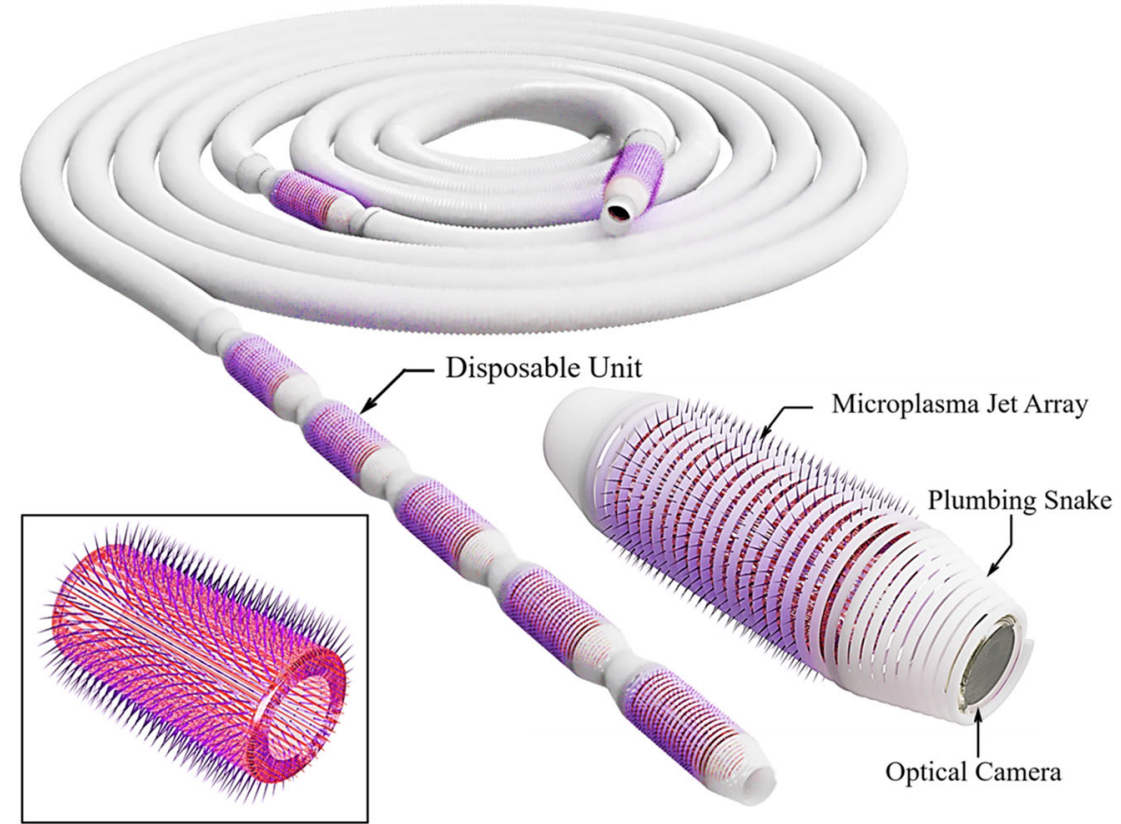

Fig. 6 Illustration of a conventional plumbing snake modified so as to incorporate cylindrical sections generating arrays of radiallypropagating plasma jets. A camera is installed at one end of the snake, and the inset is a qualitative drawing of one cylindrical microplasma array section (Ref. ${ }^{22}$ )

images were captured for each biofilm coupon. Seventy cross-sectional images were selected randomly for further analysis. Each of these images was processed by ImageJ software (http://imagej.nih.gov/ij/) so as to suppress or eliminate background noise. Also, the mean thickness of all biofilms was determined by analyzing the gray scale gradient through automatic thresholding with the MATLAB program developed by Derlov et $\mathrm{al}^{28}$

After the completion of the OCT scans, the same biofilm samples were stained following the protocol of the LIVE/DEAD BacLight ${ }^{\mathrm{TM}}$ Bacterial Viability Kit (Thermo Fisher Scientific). This assay determines the viability of the cell membrane. Living cells were stained green with SYTO 9 while dead cells were stained red (propidium iodide) for subsequent evaluation by CLSM. Images were acquired with a Leica SP8 laser scanning confocal microscope in which photoexcitation occurs at $488 \mathrm{~nm}$ ( $\mathrm{Ar}$ ion laser) and the detection wavelengths are 500 and $635 \mathrm{~nm}$ for SYTO 9 and propidium red, respectively. Final images were produced from the microscope data by LAS X software offered by Leica Microsystems, and red and green intensity maps were generated from the pixel data with ImageJ software and analysis by the MATLAB program COMSTAT.

As additional diagnostics of the microplasma treatment process, several biofilm coupons were also examined, after differing periods of microplasma exposure, by scanning electron microscopy (SEM) and energy dispersive spectroscopy (EDS).

\section{Detection of microplasma-produced reactive oxygen species}

Knowledge of the absolute concentrations of several critical oxygenbearing radicals and excited species, such as $\mathrm{OH}^{1} \mathrm{O}_{2}, \mathrm{H}_{2} \mathrm{O}_{2}$, and $\mathrm{O}_{3}$, is essential to assessing the efficacy of microplasmas for the deactivation of bacterial pathogens. To this end, tests were conducted in which a beaker $3.5 \mathrm{~mm}$ in height was situated immediately beneath the exit face of the microplasma arrays. The beaker contained a $10 \mathrm{~mL}$ solution of phosphatebuffered saline solution (PBS; $\mathrm{pH}=7.4)$ and $100 \mu \mathrm{M}$ of either phenol $(99 \%$, Acros Organics) or furfuryl alcohol (FFA; $98 \%$, Acros Organics), the latter of which serve as a diagnostic of $\mathrm{OH}$ (phenol) or ${ }^{1} \mathrm{O}_{2}$ (FFA), respectively. After the appropriate solution was exposed to the He plasma jets emerging into room air, samples were withdrawn and the decay of the desired probe was detected by liquid chromatography with an Agilent series 1200 HPLC chromatograph having an Eclipse Plus C18 $(3.5 \mu \mathrm{m})$ column. Separation was performed with water and acetonitrile with a ratio of 50:50 for phenol and 40:60 for FFA, and the flow rate and injection volume were fixed at $0.3 \mathrm{~mL} / \mathrm{min}$ and $20 \mu \mathrm{L}$, respectively. The detection wavelengths for phenol and FFA were $268 \mathrm{~nm}$ and $216 \mathrm{~nm}$, respectively.

The concentration of the designated probe was calculated on the basis of the standard curve for the same probes in $\mathrm{PBS}^{29}$. For the $\mathrm{OH}$ radicals generated by the microplasma array, the concentration in the beaker solution was determined from the decay of phenol in solution and is given by the product of the rate constant for the phenol-OH interaction $(1.4 \times$ $\left.10^{10} \mathrm{M}^{-1} \mathrm{~s}^{-1}\right)^{30}$, and the phenol concentration. Similarly, the ${ }^{1} \mathrm{O}_{2}$ concentration was determined from the product of the ${ }^{1} \mathrm{O}_{2}-\mathrm{FFA}$ interaction rate constant $\left(1.4 \times 10^{8} \mathrm{M}^{-1} \mathrm{~s}^{-1}\right)^{23,31}$, and the FFA concentration. Measurements of the hydrogen peroxide concentration were facilitated by a colorimetric test kit (Hydrogen Peroxide-CHEMets ${ }^{\circledast}$ Visual Kit). Hydrogen peroxide oxidizes ferrous iron to the ferric state, resulting in the formation of a red thiocyanate complex. By comparing the colors of the test samples with those of standard samples (provided by the test kit), the concentrations of $\mathrm{H}_{2} \mathrm{O}_{2}$ in the test samples were determined. The minimum detectable value was $1.5 \mu \mathrm{M}$. Finally, the concentration of ozone dissolved in solution was measured with a second test kit (HACH OZ-2 (2064400)). Each test was performed at least three times, in order to obtain a statistically significant result. 


\section{DATA AVAILABILITY}

All data supporting the findings of this study are available from the corresponding author upon request.

\section{ACKNOWLEDGEMENTS}

The technical assistance of Dr. Dianwen Zhang (Beckman Institute, University of Illinois) and Yulin Wang (Huaqiao University, China) with regard to the CLSM experiments, and the support of the U.S. Environmental Protection Agency EPA (agreement no. RD-83487001), and the U.S. Air Force Office of Scientific Research under grant no. FA9550-14-1-0002, as well as the National Institutes of Health under grant no. R01EB013723, are gratefully acknowledged.

\section{AUTHOR CONTRIBUTIONS}

P.P.S. was involved in the design, execution, and analysis of the experiments, as well as writing the manuscript. E.A.A. was involved in experimental design, performance, and analysis of the radical detection studies. C.H.H. was involved in the design and performance of the confocal laser scanning microscopy measurements. Y.S. was involved in experimental design, preparing biofilms, and writing the manuscript. G.L. $M$. was involved with experiment design, as well as acquring, and analyzing optical coherence tomography images. S.Y.Z. and Z.K.T. were involved in performing the plasma treatments and measuring hydrogen peroxide. S.A.B. was involved in analyzing coherence tomography images and preparing the manuscript. J.G.E., and T. H.N. were involved in experimental design, data analysis, and the preparation of the manuscript.

\section{ADDITIONAL INFORMATION}

Supplementary information accompanies the paper on the npj Biofilms and Microbiomes website (https://doi.org/10.1038/s41522-018-0063-4).

Competing interests: P.P.S. and J.G.E are listed as inventors on U.S. Patent Application No. 15/98105 (May 2018). The remaining authors declare no competing interests. J.G.E. is a co-inventor of U.S. Patent No.8,957,572.

Publisher's note: Springer Nature remains neutral with regard to jurisdictional claims in published maps and institutional affiliations.

\section{REFERENCES}

1. Lau, H. Y. \& Ashbolt, N. J. The role of biofilms and protozoa in Legionella pathogenesis: implications for drinking water. J. Appl. Microbiol. 107, 368-378 (2009).

2. Tison, D. L., Pope, I. D. H., Cherry, W. B. \& Fliermans, C. B. Growth of Legionella pneumophila in association with blue- green algae (Cyanobacteria). Appl. Environ. Microbiol. 39, 456-459 (1980).

3. Wadowsky, R. M. \& Yee, R. B. Satellite growth of Legionella pneumophila with an environmental isolate of Flavobacterium breve. Appl. Environ. Microbiol. 46, 1447-1449 (1983).

4. Stout, J. E., Yu, V. L. \& Best, M. G. Ecology of Legionella pneumophila within water distribution systems. Appl. Environ. Microbiol. 49, 221-228 (1985).

5. Temmerman, R., Vervaeren, H., Noseda, B., Boon, N. \& Verstraete, W. Necrotrophic growth of Legionella pneumophila. Appl. Environ. Microbiol. 72, 4323-4328 (2006).

6. Thomas, J. M. \& Ashbolt, N. J. Do free-living amoebae in treated drinking water systems present an emerging health risk? Environ. Sci. Technol. 45, 860-869 (2011).

7. Cargill, K. L., Pyle, B. H., Sauer, R. L. \& McFeters, G. A. Effects of culture conditions and biofilm formation on the iodine susceptibility of Legionella pneumophila. Can. J. Microbiol. 38, 423-429 (1992).

8. Cooper, I. R. \& Hanlon, G. W. Resistance of Legionella pneumophila serotype 1 biofilms to chlorine-based disinfection. J. Hosp. Infect. 74, 152-159 (2010).

9. Kotay, S., Chai, W., Guilford, W., Barry, K. \& Mathers, A. J. Spread from the sink to the patient: in situ study using green fluorescent protein (GFP) - expressing Escherichia coli to model bacterial dispersion from-washing sink-trap reservoirs. Appl. Environ. Microbiol. 83, 1-12 (2017).

10. Kim, B. R., Anderson, J. E., Mueller, S. A., Gaines, W. A. \& Kendall, A. M. Literature review-Efficacy of various disinfectants against Legionella in water systems. Water Res. 36, 4433-4444 (2002).

11. Bridier, A., Briandet, R., Thomas, V. \& Dubois-Brissonnet, F. Resistance of bacterial biofilms to disinfectants: a review. Biofouling 27, 1017-1032 (2011).
12. Becker, K. H., Schoenbach, K. H. \& Eden, J. G. Microplasmas and applications. J. Phys. D. Appl. Phys. 39, R55-R70 (2006).

13. Graves, D. B. The emerging role of reactive oxygen and nitrogen species in redox biology and some implications for plasma applications to medicine and biology. J. Phys. D. Appl. Phys. 45, 263001 (2012).

14. Pan, J. et al. Cold plasma therapy of a tooth root canal infected with Enterococcus faecalis biofilms in vitro. J. Endod. 39, 105-110 (2013).

15. Li, Y. et al. Evaluation of cold plasma treatment and safety in disinfecting 3-week root canal Enterococcus faecalis biofilm in vitro. J. Endod. 1-6 (2015). https://doi. org/10.1016/j.joen.2014.10.020.

16. Sun, Y. et al. Inactivation of Candida biofilms by non-thermal plasma and its enhancement for fungistatic effect of antifungal drugs. PLoS One 7, e40629 (2012).

17. Sun, P. et al. Atmospheric pressure cold plasma as an antifungal therapy. Appl. Phys. Lett. 98, 021501 (2011)

18. Xu, Z. et al. Inactivation effects of non-thermal atmospheric-pressure helium plasma jet on Staphylococcus aureus biofilms. Plasma Process. Polym. 12, 827-835 (2015).

19. Alshraiedeh, N. H. et al. Eradication and phenotypic tolerance of Burkholderia cenocepacia biofilms exposed to atmospheric pressure non-thermal plasma. Int. J. Antimicrob. Agents 47, 446-450 (2016).

20. Taghizadeh, L. et al. Inactivation of biofilms using a low power atmospheric pressure argon plasma jet; the role of entrained nitrogen. Plasma Process. Polym. 12, 75-81 (2014).

21. Sun, P. P., Cho, J. H., Park, C. H., Park, S. J. \& Eden, J. G. Close-packed arrays of plasma jets emanating from microchannels in a transparent polymer. IEEE Trans. Plasma Sci. 40, 2946-2950 (2012).

22. Eden, J. G. \& Sun, P. P. Microplasma devices for surface or object treatment and biofilm removal, U.S. Patent Application No. 15/98105 (2018).

23. Janjaroen, D. et al. Roles of ionic strength and biofilm roughness on adhesion kinetics of Escherichia coli onto groundwater biofilm grown on PVC surfaces. Water Res. 47, 2531-2542 (2013).

24. Shen, Y. et al. Response of simulated drinking water biofilm mechanical and structural properties to long-term disinfectant exposure. Environ. Sci. Technol. 50, 1779-1787 (2016).

25. Xi, C., Marks, D., Schlachter, S., Luo, W. \& Boppart, S. A. High-resolution threedimensional imaging of biofilm development using optical coherence tomography. J. Biomed. Opt. 11, 34001/1-6 (2006).

26. Shen, Y. et al. Role of biofilm roughness and hydrodynamic conditions in Legionella pneumophila adhesion to and detachment from simulated drinking water biofilms. Environ. Sci. Technol. 49, 4274-4282 (2015).

27. Ahmad, A. et al. Real-time in vivo computed optical interferometric tomography. Nat. Photonics 7, 444-448 (2013).

28. Derlon, N., Peter-Varbanets, M., Scheidegger, A., Pronk, W. \& Morgenroth, E. Predation influences the structure of biofilm developed on ultrafiltration membranes. Water Res. 46, 3323-3333 (2012).

29. Rosado-Lausell, S. L. et al. Roles of singlet oxygen and triplet excited state of dissolved organic matter formed by different organic matters in bacteriophage MS2 inactivation. Water Res. 47, 4869-4879 (2013).

30. Alexieva, Z., Yemendzhiev, H. \& Zlateva, P. Cresols utilization by Trametes versicolor and substrate interactions in the mixture with phenol. Biodegradation 21, 625-635 (2010).

31. Haag, W. R. \& Hoigne, J. A. Singlet oxygen in surface waters. 3. photochemical formation and steady-state concentrations in various types of waters. Environ. Sci. Technol. 20, 341-348 (1986).

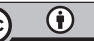

Open Access This article is licensed under a Creative Commons Attribution 4.0 International License, which permits use, sharing, adaptation, distribution and reproduction in any medium or format, as long as you give appropriate credit to the original author(s) and the source, provide a link to the Creative Commons license, and indicate if changes were made. The images or other third party material in this article are included in the article's Creative Commons license, unless indicated otherwise in a credit line to the material. If material is not included in the article's Creative Commons license and your intended use is not permitted by statutory regulation or exceeds the permitted use, you will need to obtain permission directly from the copyright holder. To view a copy of this license, visit http://creativecommons. org/licenses/by/4.0/.

(c) The Author(s) 2018 